\title{
Costing a Community Based Screening Programme for the Detection of Glaucoma
}

\author{
S. J. JONES, ${ }^{1}$ S. A. VERNON,${ }^{2}$ L. CATER,${ }^{3}$ D. J. HENRY ${ }^{1}$ \\ Nottingham
}

\begin{abstract}
Summary
The cost of detecting a case of glaucoma in a community based screening programme was calculated using data from a pilot study on a population aged 50 and over.

The cost per screen was estimated at $£ 3.35$ with a total cost of $£ 311$ per case detected. These estimates include the cost of both the algorithmically based screening programme and the hospital assessment of true and false positives.

These costs are considered to compare favourably with the potential costs incurred by paying optometrists to screen high risk groups whilst providing the opportunity to detect over twice the number of cases of occult glaucoma.
\end{abstract}

Glaucoma is a common but preventable cause of blindness. ${ }^{1}$ Ophthalmologists treat some patients with raised intraocular pressure (IOP) whether or not field loss is apparent if significant risk factors are present. ${ }^{2}$ Many patients are referred to specialist eye services with advanced disease ${ }^{3}$ initial detection often being by opticians. ${ }^{4,5}$ The ratio of known cases to occult cases in the community remains at one to one ${ }^{6}$ a figure that has remained unchanged since the 1960s. This indicates that little, if any advance has been made in the detection of early disease and that conventional methods of detecting glaucoma are unsatisfactory.

Population screening may be worthwhile depending on the sensitivity and specificity of the tests used and the cost involved. Utilising data from a large number of studies, Gottlieb and his colleagues evaluated methods of screening for glaucoma and concluded that it is probably only cost effective when targeted at sub groups of the population. ${ }^{7}$ However, they failed to consider the costs of identifying these groups and were also unable to examine the possibility of undertaking screening within a primary care setting, such as exists in the United Kingdom. We have previously reported the results of a feasibility study of screening in general practice. ${ }^{6}$ This study incorporated measurement of intraocular pressure, visual field analysis and optic disc assessment to reduce false negatives to a minimum. We propose that intraocular pressures measured with a Pulsair non contact tonometer as an adequate first line screening test.

This paper considers the cost of running a general practice based screening programme for those over 50 years of age on a routine basis.

\section{Patients and Methods}

The screening procedure costed is shown in Figure 1. IOPs would be measured with a Keeler Pulsair non-contact tonometer, using the mean of four pulses per eye. Patients requiring visual field analysis would undergo a

${ }^{1}$ Department of General Practice, ${ }^{2}$ Academic Unit of Ophthalmology, ${ }^{3}$ Practice Nurse.

Correspondence to: S. J. Jones M.Phil. Dept of General Practice, University Hospital, Nottingham NG7 2UH. 

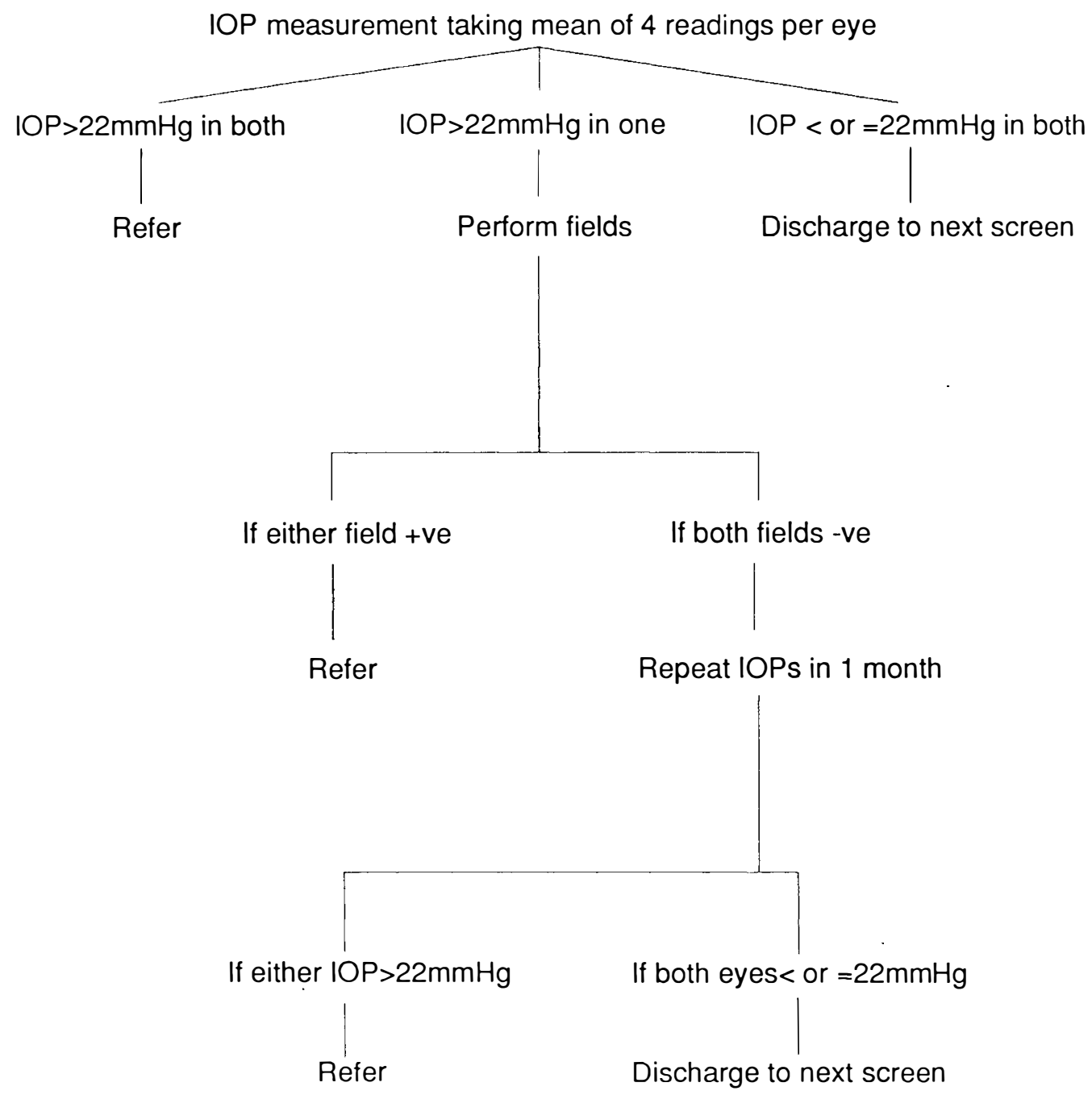

Fig. 1. Algorithm used for costing of Community Screening Programme.

strict field protocol such as the one used in the pilot study. It was assumed that half of the patients requiring a second IOP screen with normal fields would maintain a high pressure and be referred to an eye outpatient department, the rest being regarded as normal. Initial screening would take place in general practices undertaken by a nurse supported by a clerk. The nurse would undertake the screening procedure and the clerk would be responsible for sending out invitations and following up non attenders. The screening could be done by a trained technician but for the purposes of this exercise, it was assumed that a nurse (Grade $G$ ) would be suitable.
The time taken for each test, including explanation, was measured, as was that involved in identifying patients, sending out invitations and organising screening sessions. These times were used to estimate the total population that could be screened per annum if a full time trained nurse and clerk were employed. A non-attendance rate of $20 \%$ was used in the calculations. Half the nurse's time was assumed to be spent on initial screening, the rest on performing fields, second stage IOP screening and organisation. The screening programme would be under the overall supervision of a consultant ophthalmologist and the time necessary for this was estimated. 
Staff costs were based on the mid point of the relevant salary scale with additions for insurance and superannuation. The costs of a hospital outpatient assessment for those requiring one were included as part of the screening programme. The cost of an outpatient visit was estimated on the basis of average medical, nursing and clerical staff costs per outpatient visit obtained from Health Service costing returns. ${ }^{8}$

Postage and stationery costs were included, these were mainly for sending out invitations and reminder letters. It was assumed that both nurse and clerk would be based in the eye outpatient department but would travel from practice to practice. Annual costs of the Pulsair non contact tonometer and Henson CFS 2000 field screener were calculated from list prices. Both items of equipment were assumed to have a life span of five years and costs were discounted at $4 \%$ per annum.

An additional 30\% was added to all estimated costs for overheads, this figure being chosen as reflecting other Health Service costs, but it is probably an overestimate for a peripatetic service such as this.

\section{Results}

Using the Pulsair tonometer, eight measures of intraocular pressure can be taken in an average of two minutes. ${ }^{6}$ The average total time per screen, including explanations to the patient, was estimated at three minutes. Thus, a full time nurse was estimated to be able to hold screening sessions for 12,500 patients per annum. On the basis of a 20 per cent non attendance rate, 10,000 would actually be screened. On the basis of the pilot study, 126 true cases of glaucoma would be expected to be identified in such a population. A further 206 patients would also have raised pressures at their second screen and be referred to the Eye Outpatient Department but be classified as false positives.

The total cost of an outpatient visit was estimated at $£ 17.33$. This figure included medical staff costs of $£ 6.02$, nursing staff costs of $£ 2.95$, equipment and drugs costs of $0.50 \mathrm{p}$ and $£ 7.86$ for general services. We assumed screening for glaucoma to be necessary every five years and the annual costs of screening are shown in Table I. It should be noted that the total annual costs include the cost of hospital outpatient review of patients with abnormal results. The total Health Service cost per patient screened therefore amounts to $£ 3.35$, with a cost of $£ 311.00$ per case of treatable glaucoma detected.

In a five year time period the nurse and clerk team would be able to invite 62,500 individuals aged 50 years and over for screening. Assuming that 50,000 attended then a total of 1660 patients would have positive screens of whom 630 would require treatment.

\section{Discussion}

Ten per cent of patients with glaucoma are registerable as blind on presentation. ${ }^{3}$ The rationale for screening for glaucoma is that early detection and prompt treatment will prevent or delay the onset of visual loss. This has obvious benefits for the patients concerned, who will retain a better quality of life. The prevention of blindness also has benefits to society as a whole. Patients live approximately ten years following blind registration for glaucoma, ${ }^{9}$ and glaucoma accounts for 14 per cent of blind registrations in Nottingham. ${ }^{10}$ As treatment can prevent or delay blindness, major savings would be expected in the provision of special care, including the adaptation of housing, the provision of special aids and in tax deductions. Although measurement of these costs was not

Table I Annual costs of a general practice based screening programme (10,000 screens per year).

\begin{tabular}{lr}
\hline Unit & $\begin{array}{c}\text { Costs in pounds } \\
\text { per year }\end{array}$ \\
\hline Staff & 14662 \\
$\quad$ Nurse & 5996 \\
Clerk & 1000 \\
$\quad$ Consultant supervision & \\
Equipment & 721 \\
$\quad$ Pulsair Noncontact Tonometer & 721 \\
$\quad$ Henson CFS 2000 Field Analyser & 140 \\
Sundries & 2530 \\
$\quad$ Travel & 7731 \\
Postage and Stationery & \\
$\quad$ General Services & 2184 \\
Hospital outpatient review & 3570 \\
$\quad$ True positive cases & 39255 \\
\hline False positive cases & \\
\hline Total & \\
\hline
\end{tabular}


attempted in this study, they are thought to be significantly greater than the estimated cost per case detected.

The importance of early detection of glaucoma has been acknowledged by the government provision of free eye tests by an optometrist for those with recognised risk factors. From April 11989 only diabetics and those with a positive family history of glaucoma will be entitled to such a test on medical grounds. Whilst these individuals are at greater risk of developing glaucoma, only $42 \%$ of cases detected in our pilot study had either of these risk factors. ${ }^{6}$

The fee paid to optometrists for screening such individuals is $£ 10.70$, considerably more than the estimated $£ 3.55$ per screen if population screening were introduced, although only $7 \%$ of the population would be screened. If optometrists could achieve similar sensitivity and specificity results to those found in our screening study, the minimum cost per case detected would be $£ 201.00$. This does not include a costing for the identification of high risk individuals, which may be difficult and therefore substantial.

Detecting glaucoma in the higher risk groups using optometrists may, therefore, cost as much or even more per case detected, particularly as optometrist specificities estimated from recent studies vary considerably. ${ }^{4.11}$ Even if it proves to be relatively cheaper, consideration needs to be given to the relative costs of detecting all cases rather than those simply occurring in high risk groups.

In our feasibility study $90 \%$ of patients requiring treatment were detected simply using measurement of intraocular pressures, with field analysis only if IOP exceeded $22 \mathrm{mmHg}$.

An argument against tonometry as the primary screening tool in chronic simple glaucoma (CSG) is the reported low sensitivity of the method. For example, Hollows and Graham found that only $50 \%$ of individuals with classical CSG detected in a screening study had IOPs $>21 \mathrm{mmHg}$ initially. ${ }^{12}$ However, no mention is made of the maximum IOP in these individuals.

The importance of value for money has been emphasised by a recent editorial on screening. ${ }^{13}$ The risk of development or progression of field loss increases as presenting IOP rises in $\mathrm{CSG}^{14}$ and the higher the IOP, the more aggressive the glaucoma. ${ }^{15}$ Therefore, there will be a greater cost/benefit ratio in identifying subjects with the higher IOPs, particularly as treatment at present concentrates on lowering IOP.

Applying the results of our pilot study, ${ }^{6}$ we calculate that the addition of routine field analysis to the protocol, without experienced fundal assessment, increases the cost per case detected to $£ 415$, an increase of $33 \%$. This is mainly as a result of increased false positives requiring hospital assessment.

Alternatively, employing a staff grade ophthalmologist or optometrist as part of the screening programme to perform disc assessment, was estimated to increase the Health Service cost per case detected by approximately $£ 150.00$, an increase of almost $50 \%$.

We believe the cost per case detected as calculated in this study ( $£ 311)$ is not so prohibitively high to rule out community screening for glaucoma. Generous timings for the organisation of the programme were included when estimating the costs in this study. Such a programme should not be implemented, however, without considering the consequences to the Hospital Eye Service. The initial diagnostic visit is included in this costing and there would be a reduction in referrals to ophthalmic clinics for 'query glaucoma', which presently run at $14 \%$ of all referrals to a general ophthalmologist. ${ }^{11}$ This must be set against the extra workload involved in managing the occult cases detected by the screening programme.

The development of a screening programme on a trial basis is necessary to determine its feasibility across a range of general practices. The present population involved in this study is not atypical but it may be unrealistic to expect the high response achieved from patients in all areas. For example, inner city practices have notoriously low levels of attendance for screening clinics.

In order to determine whether community screening on a nationwide scale would be efficient in preventing visual deficit from glaucoma, a carefully audited study is required. It must include sufficient individuals in matched groups to ensure a statistically significant 
result. We are at present designing such a study.

The authors have no financial interest in any of the screening equipment or Keeler Ltd.

\section{References}

${ }^{1}$ Pitts Crick R. Chronic Glaucoma-A preventable cause of blindness Lancet 1974, 1: 205-7.

${ }^{2}$ Mills KB. Ocular Hypertension Res. Clin. Forums 1985, 7: 81-4.

${ }^{3}$ Elkington AR, Lewry J, MacKean J, Sargent P. AA collaborative hospital glaucoma survey. Res. Clin. Forums. 1982, 4: 31-40.

${ }^{4}$ Brittain GPH, Austin DJ, Kelly SP. A prospective study to determine sources and diagnostic accuracy of glaucoma referrals. Health Trends 1988, 20: 43-44.

${ }^{5}$ MacKean JM and Elkington AR. Referral routes to hospital of patients with chronic open angle glaucoma. Br. Med. J. 1982, 285: 1093-5.

${ }^{6}$ Vernon SA, Henry DJ, Cater L, Jones S. Screening for glaucoma in the Community by non-ophthalmically trained staff using semi-automated equipment. Eye 1990 (in Press).

${ }^{7}$ Gottlieb LK, Schwartz B, Pauker SG. Glaucoma screening: a cost-effective analysis. Surv. Ophthalmol 1983, 28: 206-26.

${ }^{8}$ Department of Health and Social Security. Health Service Costing Returns. London HMSO, 1987.

${ }^{9}$ Green JS, Bear JC, Johnson GJ. The burden of genetically determined disease. Br. J. Ophthalmol 1986, 70: 696-9.

${ }^{10}$ Aclimandos WA and Galloway NR. Blindness in the city of Nottingham (1980-1985). Eye 1988, 2: $431-4$.

${ }^{11}$ Harrison RJ, Wild JM, Hobley AJ. Referral patterns to an ophthalmic outpatient clinic by general practitioners and ophthalmic opticians and the role of these professionals in screening for ocular disease. Br. Med. J. 1988, 297: 1162-7.

${ }^{12}$ Hollows FC and Graham PA. Intraocular pressure, glaucoma and glaucoma suspects in a defined population. Br. J. Opthalmol 1966, 50: 570-86.

${ }^{13}$ Khaw KT. Screening: the need for a balance. Eye 1989, 3 (4); vii-ix.

${ }^{14}$ Kass MA, Kolker AE. Becker B. Prognostic factors in glaucomatous visual field loss. Arch. Ophthalmol 1976, 94: 1274-6.

${ }^{15}$ Grant WM and Burke JF. Why do some people go blind from glaucoma? Ophthalmology 1982, 89: 991-8. 